\title{
Monitoring UT1 from astro-geodetic techniques at the EOP Center of the IERS
}

\author{
D. Gambis and C. Bizouard
}

Observatoire de Paris, 61 avenue de l'Observatoire, Paris, France

\begin{abstract}
Monitoring the Earth rotation is essential in various domains linked to reference frames firstly with applications in orbit determination, space geodesy or Astronomy. Secondly for geophysical studies where are involved mass motions within the different external fluid layers, atmosphere, hydrosphere, core and mantle of the earth, this on time scales ranging from a few hours to decades. The Earth Orientation Centre of the IERS is continuously monitoring the earth orientation variations from results derived from the various astro-geodetic techniques. It has in particular the task of deriving an optimal combined series of UT1 which is now based mainly on Very Long Baseline Interferometry (VLBI) with some contribution of LOD derived from GPS. We give here a brief summary concerning the contribution of the various techniques to UT1 and in aprticular how the use of LOD derived from GPS can improve the combination. More details are available in Gambis (2004) and Bizouard and Gambis (2009) and the website http://hpiers.obspm.fr/eop-pc/
\end{abstract}

Keywords. Universal Time UT1, Earth Rotation

\section{UT1 determination: monitoring and accuracy}

Earth Orientation Parameters describe the orientation of the earth with respect to a non rotating reference frame. One of the parameter, Universal Time UT1 represents the rotation of the Earth around its axis. Until the 1970's, UT1 was exclusively monitored by astrometric techniques based on optical instruments like photozenithal tubes, PZT, meridian refractors and astrolabes. In the 1970's the emergence of Lunar Laser Ranging (LLR) allowed to determine UT0. In 1985 its accuracy was in the range of $0.400 \mathrm{~ms}$. Meanwhile VLBI technique was emerging and determined UT1 with an accuracy at least ten times better than LLR (Table 1).

Table 1. Contribution of astro-geodetic technique to the determination of UT1 and LOD

\begin{tabular}{|c|c|c|c|c|}
\hline Technique & Since & EOP & Time resolution & Accuracy \\
\hline ASTROMETRY & 1899 & UT1 & 5 days & $1 \mathrm{~ms}$ \\
\hline LLR & 1969 & UT0 & 1 day & $0.4 \mathrm{~ms}$ \\
\hline SLR & 1976 & LOD & 3 days & $200 \mu \mathrm{s}$ \\
\hline VLBI & $\begin{array}{l}1981 \\
1981 \\
1981\end{array}$ & $\begin{array}{r}\text { UT1 Standard } \\
\text { UT1 Intensive } \\
\text { LOD }\end{array}$ & $\begin{array}{l}3-4 \text { days } \\
1 \text { day } \\
3-4 \text { days }\end{array}$ & $\begin{array}{r}5 \mu \mathrm{s} \\
15 \mu \mathrm{s} \\
15 \mu \mathrm{s}\end{array}$ \\
\hline GPS & 1993 & LOD & 1 day & $10 \mu \mathrm{s}$ \\
\hline
\end{tabular}




\section{Contribution of LOD derived from GPS estimates in UT1 series, method of combined smoothing}

VLBI, as the only technique referring to a non rotating celestial reference frame is the main contributor of UT1. Alternatively, satellites techniques, like GPS which are realizing their celestial frame through the orbit determination do not allow determining an accurate UT1; because of mis-modelling of various perturbations, the orbit is affected by long-term systematic variations. GPS techniques can nevertheless determine daily LOD estimates of which values are slightly biased. LOD(GPS) can be used for UT1 computation when calibrated by UT1 derived from VLBI using the so-called method of "Combined Smoothing" Vondrak et al., 1999; Vondrak, 2000. Figure 1 shows the comparison of various combined UT1 series with the external Final Bulletin A series. It appears that the contribution of GPS LOD either by the direct integration of LOD(GPS) or when applying the Combined Smoothing leads to an small improvement of a few $\mu \mathrm{s}$ in the WRMS compared to the solution which does not incorporate any LOD(GPS) data. It is also striking that the contribution of intensive session is only a few $\mu$ s.

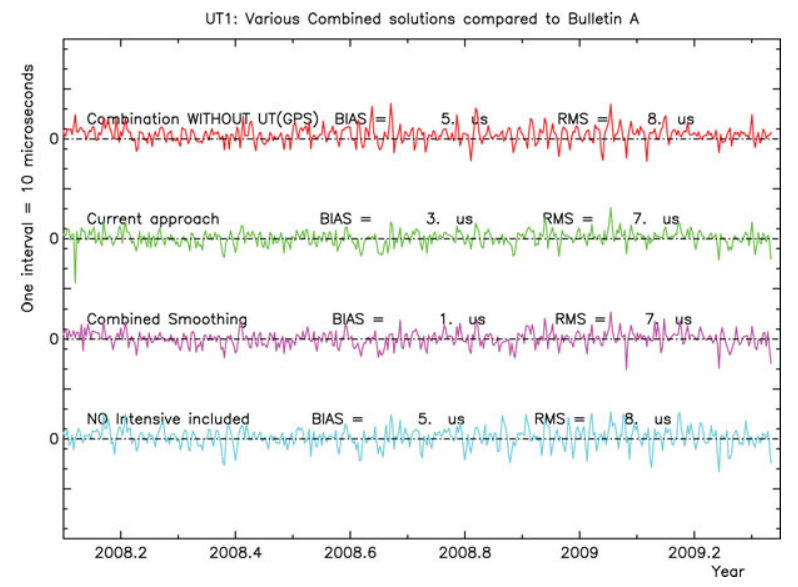

Figure 1. Comparisons of different UT1 combined series using or not UT(GPS). It appears that the contribution of GPS LOD either by the direct integration of LOD(GPS) referred as Current approach, or when applying the Combined Smoothing leads to an small improvement in the WRMS compared to the usual solution. We can remark that the inclusion of Intensive sessions does not significantly improve the final combined UT1 solution.

\section{References}

Bizouard, C. \& Gambis, D., 2009, the combined solution C04 for Earth Orientation Parameters, recent improvements, Springer Verlag series, Series International Association of Geodesy Symposia, Vol. 134, Hermann (Ed.), pp. 265-270

Gambis, D., 2004, Monitoring Earth orientation using space-geodetic techniques: state-of-theart and prospective, J. of Geodesy, Volume 78, Issue 4-5, pp. 295-303, doi 10.1007/s001900040394-1.

Vondrak, J. \& Gambis, D., 1999, Accuracy of Earth orientation parameters obtained by different techniques in different frequency windows, in: Soffel, M., Capitaine, N. (eds.) JSR 1999 Systmes de rfrence spatio-temporels and IX. Lohrmann Colloquium, Observatoire de Paris, 206-213.

Vondrak, J. \& Cepek, A., 2000, Combined smoothing method and its use in combining Earth orientation parameters measured by space techniques, Astron. Astrophys. Suppl. Ser. 147, $347-359$ 